\title{
Intraductal tubulopapillary neoplasms of the pancreas and biliary tract: The black swan of hepatobiliary surgery
}

\author{
Jilyan Decker ${ }^{1}$, Amanda Cavanaugh'1, Megan Brown², Saverio Ligato², Oscar Kenneth Serrano ${ }^{1,3}$ \\ 'Department of Surgery, University of Connecticut School of Medicine, Farmington, CT, United States, \\ ${ }^{2}$ Department of Pathology and Laboratory Medicine, Hartford Hospital, Hartford, CT, United States, \\ ${ }^{3}$ Hartford Hospital Transplant Program \& Comprehensive Liver Center, Hartford, CT, United States
}

\begin{abstract}
Intraductal tubulopapillary neoplasms (ITPNs) of the pancreas and biliary tract are rare pre-malignant entities of the biliary tract and pancreas that are difficult to diagnose preoperatively. While there are imaging characteristics that can differentiate these lesions from more common entities like adenocarcinoma or intraductal papillary mucinous neoplasms (IPMN), ITPNs are not always distinctive. Herein we present two cases of ITPN, one of biliary and the other of pancreatic origin, which had a preoperative diagnosis of cholangiocarcinoma and IPMN, respectively. We discuss our findings in these cases, patient presentation and course, review the radiographic and pathologic findings, and propose a more effective approach to the preoperative workup and diagnosis of ITPN based on our review of the contemporary literature.
\end{abstract}

Key Words: Pancreatic intraductal neoplasms; Bile duct neoplasms

\section{INTRODUCTION}

Intraductal tubulopapillary neoplasms (ITPNs) of the pancreas and bile duct represent rare premalignant neoplasms that were first recognized by the World Health Organization (WHO) in 2010 [1]. The first case of ITPN in the pancreas (p-ITPN) was described in 1992 by Shahinian et al. [2], while the first biliary ITPN (b-ITPN) case was reported in 2010 by Park et al. [3]. Typically, ITPN presents with jaundice, abdominal pain, and weight loss, resembling pancreatic adenocarcinoma or cholangiocarcinoma, and is most commonly found in patients over 50 years old, with a female overrepresentation. ITPN represents less than $1 \%$ of all pancreatic exocrine neoplasms, $3 \%$ of pancreatic intraductal neoplasms [4], and $15 \%$ of

Received: January 31, 2021, Revised: May 30, 2021,

Accepted: June 2, 2021

Corresponding author: Oscar Kenneth Serrano

Hartford Hospital Transplant Program \& Comprehensive Liver Center,

85 Seymour Street, Suite 320, Hartford, CT 06106, United States

Tel: +1-860-972-4219, Fax: +1-860-549-1476,

E-mail: Oscar.Serrano@hhchealth.org

ORCID: https://orcid.org/0000-0001-5076-0938

opyright (C) The Korean Association of Hepato-Biliary-Pancreatic Surgery intraductal neoplasms of the bile duct [5]. What distinguishes ITPN from other intraductal neoplasms, such as intraductal papillary mucinous neoplasms (IPMN), is their uniquely favorable prognosis.

Given the obscurity of these lesions and the heterogeneity of presentation, we report two cases of ITPN from our center with unusual clinical findings and review the literature on this rare entity.

\section{CASES}

\section{Pancreatic intraductal tubulopapillary neoplasm}

A 76-year-old male with a previous history of coarctation repair as a child, followed by triple bypass with aortic valve replacement, and eventually a heart transplant, presented to the emergency room with a complaint of severe diffuse abdominal pain located in the upper quadrants. The pain had been present for about a week at the time of his presentation. He reported associated nausea without vomiting. He did not have any jaundice or change in the color of his urine or stools. He did not report any weight loss or recent change in appetite. In the emergency room, a computed tomography (CT) scan was performed, which demonstrated a dilated pancreatic duct measuring $9 \mathrm{~mm}$, a common bile duct measuring $1.3 \mathrm{~cm}$, and a 1.3 $\mathrm{cm} \times 1.3 \mathrm{~cm}$ hypodense area in the pancreatic head described 
as a cystic neoplasm (Fig. 1A). He was then transferred to our institution for further care and evaluation of this newly found mass. Magnetic resonance imaging (MRI) revealed a pancreatic duct diffusely dilated to $1.3 \mathrm{~cm}$, and a $3-\mathrm{cm}$ mass in the head of the pancreas without vessel involvement (Fig. 1B, 1C). Subsequently, an endoscopic retrograde cholangiopancreatography (ERCP) with endoscopic ultrasound (EUS) was performed and the fine needle aspirate results were consistent with IPMN. He had a pancreaticoduodenectomy for a presumed diagnosis of main duct IPMN. An intraoperative frozen section of the pan- creatic neck was consistent with IPMN and mild atypia.

The pancreatoduodenectomy specimen was bivalved to reveal a tan solid mass within the pancreatic duct (Fig. 2A), with no cystic structure or associated mucin identified. The lesion completely occluded the lumen and measured $3.5 \mathrm{~cm}$ longitudinally within the pancreatic duct. Histological low-power microscopic examination showed an intraductal lesion with tubulopapillary architecture and conspicuously, no mucin production (Fig. 2B, 3C). The high-power evaluation showed highgrade nuclei atypia and immunohistochemical findings typical
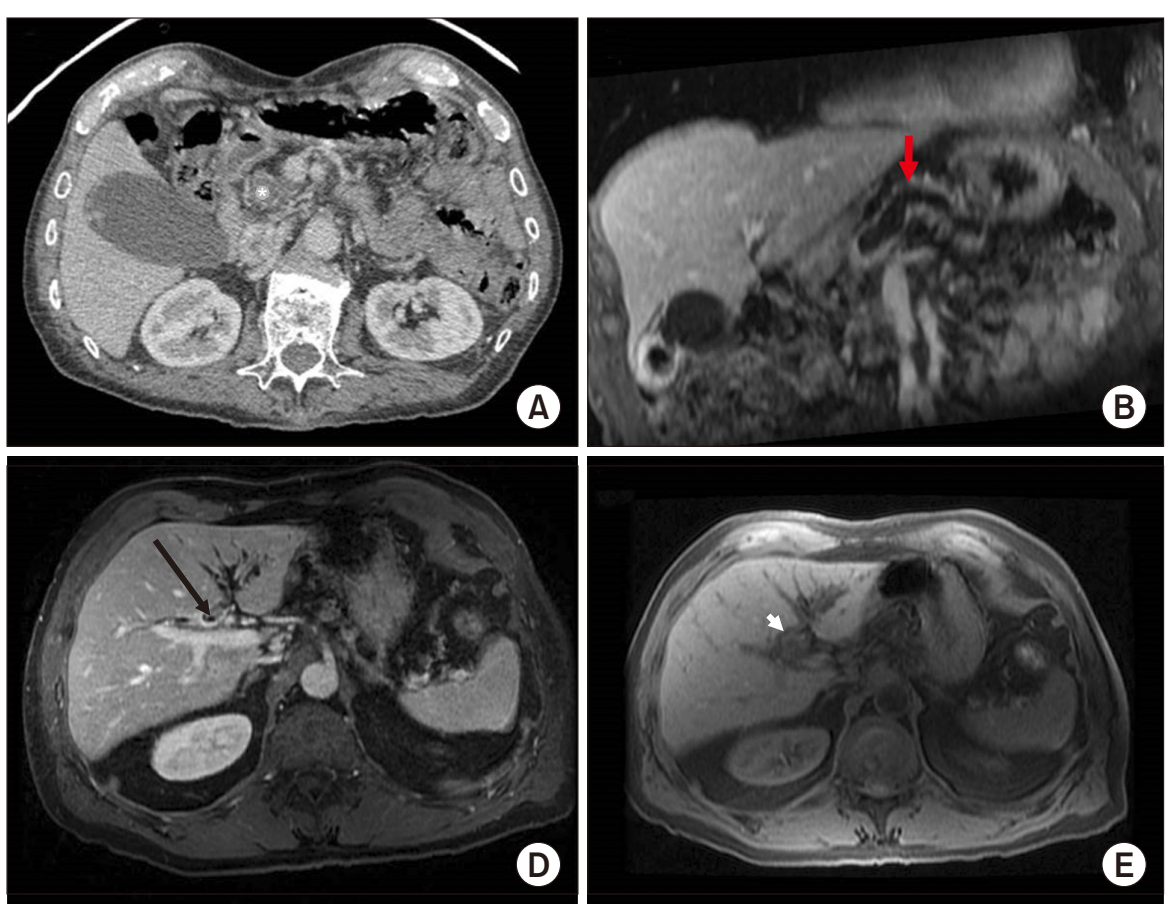
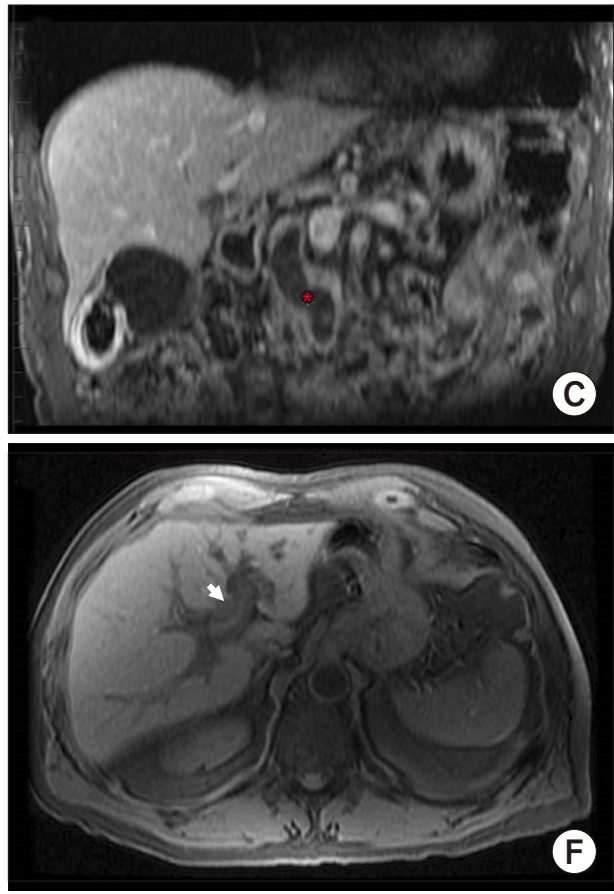

Fig. 1. (A) Cross-sectional computed tomography (CT) imaging demonstrating an atrophic pancreas with a cystic neoplasm in the pancreatic head, measuring $1.3 \mathrm{~cm} \times 1.3 \mathrm{~cm}$ (white asterisk). (B) Coronal magnetic resonance imaging (MRI) imaging demonstrated a prominent dilated pancreatic duct, measuring $9 \mathrm{~mm}$, which tapered in caliber at the pancreatic head (arrow). (C) Additionally, the proximal dilated intrapancreatic duct demonstrated increased T1 and decreased T2 signals within the duct in the region of the dilated pancreatic head (red asterisk), suggesting debris or mucin, with more simple-appearing T2 bright fluid in the more proximal duct. (D) Cross-sectional CT imaging demonstrating significant biliary dilatation, particularly of the left lobe intrahepatic biliary radicles. At the level of the common hepatic duct upstream from the cystic duct insertion is a circumferential mass (black arrow). (E) Cross-sectional MRI imagining confirmed a circumferential mass, which was hyper-enhanced (white arrowhead) and retained contrast on delayed imaging, consistent with cholangiocarcinoma. (F) The mass (white arrowhead) measured up to $11 \mathrm{~mm}$ in diameter and extended to the hepatic hilum where it extended into the left hepatic duct just beyond the secondary to a biliary radical duct.
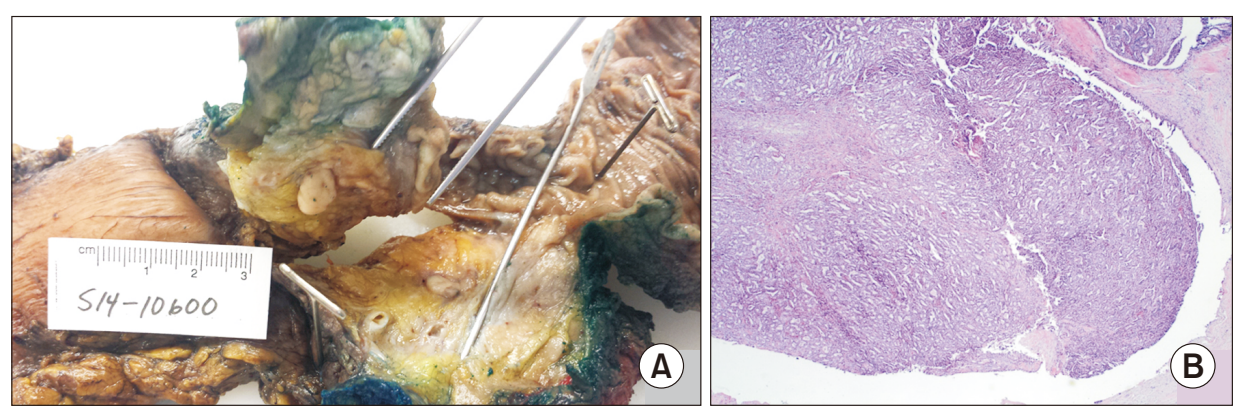

Fig. 2. (A) Whipple specimen showing intraductal tubulopapillary neoplasm (ITPN) of the pancreas within the common pancreatic duct. (B) Low-power microscopic view of H\&E-stained slide at $20 \times$ magnification showing ITPN filling the pancreatic duct (duct lining). 

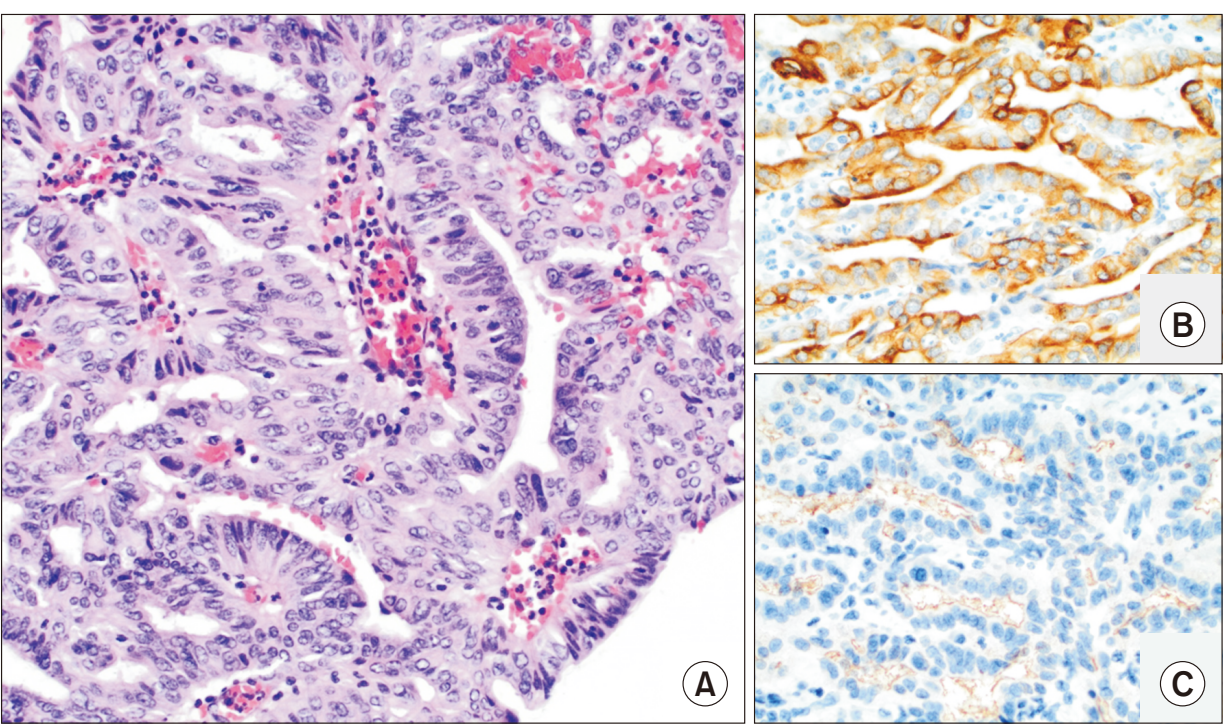

Fig. 3. (A) High-power microscopic image of H\&E-stained slide (400x magnification) showing tubular architecture and high-grade nuclear features with an absence of mucin production. (B) Immunohistochemical staining for $\mathrm{CK} 7$ (200x magnification) showing positive membranous and cytoplasmic expression. (C) Immunohistochemical stain for MUC1 (200x magnification) showing membranous luminal expression. of ITPN of the pancreas. The lesion was found to be positive for cytokeratins 7 (Fig. 3B) and 19, and CA19-9. Neuroendocrine markers synaptophysin, chromogranin, beta-catenin, carcinoembryonic antigen (CEA), MUC5AC, and p53 were all negative. MUC1 showed luminal expression (Fig. 3C). The typical immunohistochemical findings for ITPN of the pancreas include positive MUC1, cytokeratin 7, and/or cytokeratin 19, in addition to negative trypsin, MUC2, MUC5AC, and fascin [4].

His postoperative course was complicated by the development of an ileus, which eventually resolved, and he was discharged to home without further complications. Since his surgery, he has been followed with yearly MRIs and has remained disease-free. It is important to note that the literature does not favor MRI over CT for surveillance imaging of the pancreas. In fact, several studies in the literature suggest the non-inferiority of CT over MRI [6]. However, our center prefers pancreatic imaging in the remnant pancreas with MRI since some studies suggest a higher sensitivity and reader confidence in MRI for the detection of individual morphologic features such as duct communication compared to CT $[7,8]$.

\section{Biliary intraductal tubulopapillary neoplasm}

A 68-year-old male with a history of hypertension was in his usual state of health when he noticed his urine had turned orange, his stool clay-colored, and he was experiencing intermittent epigastric pain. He also had a recent unintentional $7 \mathrm{~kg}$ (15 1b) weight loss in the prior six weeks. He was a former smoker, having quit 10 years ago. He had no significant alcohol or illicit substance use history. He had a paternal family history of alcoholism and unspecified cancer in his brother. He was first seen by his primary care physician, at which time, blood work showed elevated liver function test values. A CT scan of the abdomen showed dilation of the intrahepatic ducts and rounded, elongated density in the common hepatic duct measuring 13 $\mathrm{mm}$ in diameter (Fig. 1D). There was no downstream ductal dilation. He was referred to a gastroenterologist, who performed an ERCP, which showed malignant-appearing stenoses in the common bile duct. A sphincterotomy was performed and a stent was placed in the left hepatic duct. Intraductal brushings showed occasional groups of bile duct epithelium with prominent nucleoli and nuclear crowding and overlap consistent with adenocarcinoma, presumed to be cholangiocarcinoma.

Further preoperative imaging included magnetic resonance cholangiopancreatography (MRCP), which showed a Bismuth IIIB lesion. Tumor markers CEA and CA19-9 were normal. He was taken to the operating room and had a left hepatectomy with bile duct resection and Roux-en-Y hepaticojejunostomy. His postoperative course was uneventful, and he left the hospital shortly after regaining bowel function on postoperative day six.

His final pathology demonstrated a b-ITPN with high-grade dysplasia measuring $3.5 \mathrm{~cm}$ within the lumen of the left hepatic duct. All margins were negative. The remaining liver parenchyma showed cholestasis indicative of mechanical obstruction. The hepatectomy specimen revealed a tan, solid, and irregular polypoid lesion within a bifurcation of the hepatic duct, which measured $3.5 \mathrm{~cm}$ (Fig. 4A). The lesion was entirely contained within the duct with a focal area of attachment identified and no associated mucin. Low-power histologic examination revealed tubulopapillary architecture and the absence of mucin production (Fig. 4B, 4C). High-power microscopic examination showed high-grade nuclear atypia without an invasive component or necrosis and the immunohistochemical findings were consistent with b-ITPN (Fig. 5A, 5B). The lesion demonstrated strong positivity for cytokeratin 7 , luminal expression for MUC1 (Fig. 5C, 5D), and patchy positivity for IMP3 and an albumin probe in in situ hybridization. Focal and weak positivity was observed for MUC5AC. The expression of MUC6, 

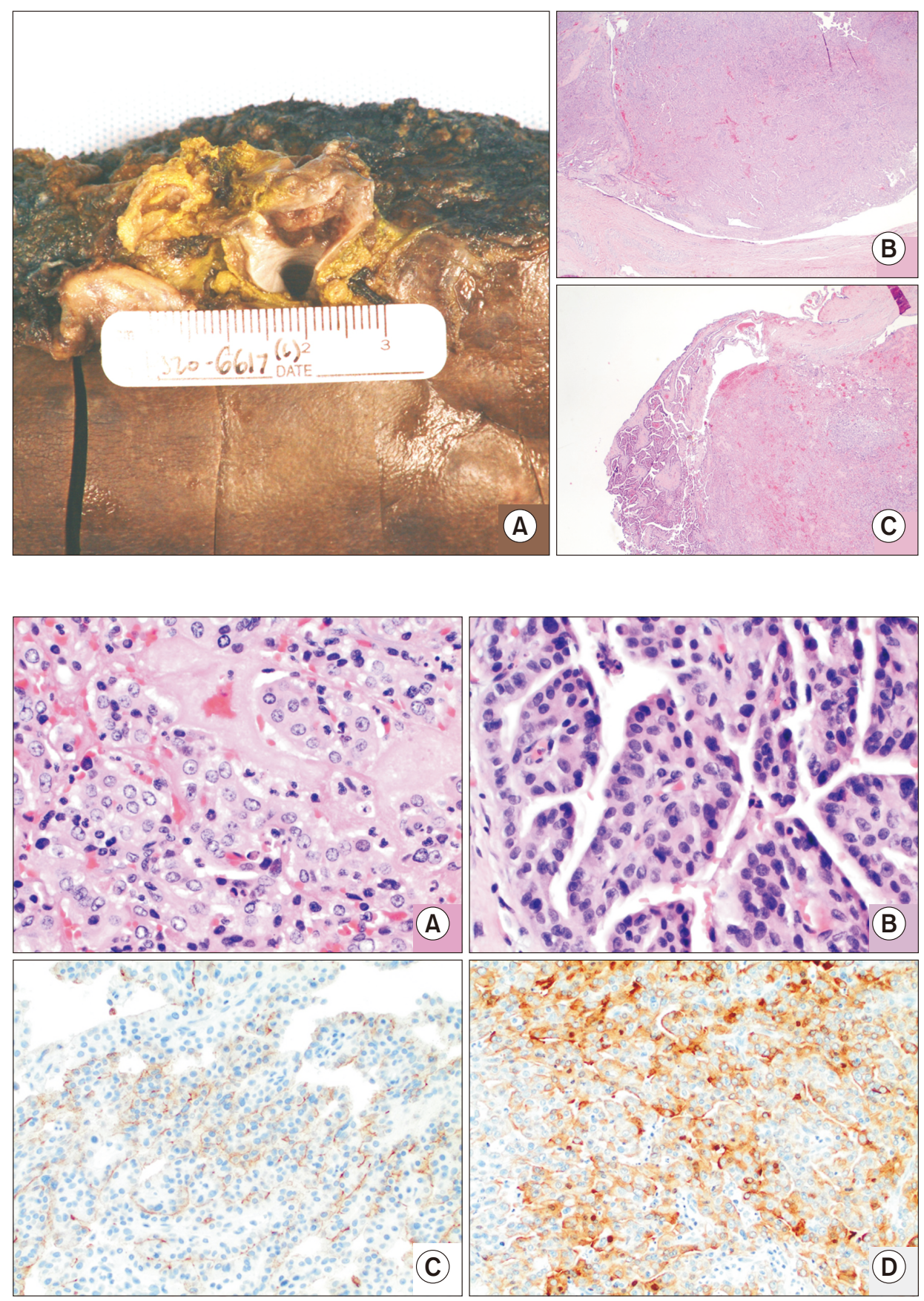

Fig. 4. (A) Resected partial hepatectomy specimen showing intraductal tubulopapillary neoplasm (ITPN) in the left hepatic duct. (B, C) Low-power microscopic examination of H\&E-stained slide at 20x magnification showing ITPN filling the left hepatic duct (duct lining), with solid and papillary architecture, and a focally attached stalk.
MUC2, HepPar-1, CK20, and CDX2 in the lesion was negative. The immunohistochemical expression in b-ITPN has been observed to have characteristics similar to those of p-ITPN, and include the positive expression of MUC1 and MUC6, and the absence of MUC2 and MUC5AC. b-ITPN has also been reported to have positive expressions of CK19 and CA19-9, while synaptophysin, chromogranin, and CA-125 were negative in all cases [4]. In this case, a next-generation sequencing (NGS)

solid tumor combined variant plex and RNA fusion panel was performed, and no DNA variants or RNA fusions were detected.

At a multidisciplinary tumor board, curative surgery was decided and the patient did not require any further treatment. $\mathrm{He}$ will be followed every three months for two years to evaluate any potential recurrence.
Fig. 5. (A) High-power microscopic image of H\&E-stained slide at $400 \times$ magnification, which shows tubular architecture and high-grade nuclear features, and papillary architecture with fibrovascular cores, high nuclear grade, and an absence of mucin production. (B, C) Immunohistochemical stain for MUC1 (B: $400 \times$ magnification, C: $200 \times$ magnification) showing membranous luminal expression. (D) Immunohistochemical stain for CK7 (200x magnification) showing positive membranous and cytoplasmic expression. 


\section{DISCUSSION}

ITPN is a rare premalignant entity that is often not diagnosed until after surgical resection. As presented herein, preoperative radiologic, endoscopic, and biopsy studies have proven unreliable for a definitive diagnosis. Standardized guidelines are difficult to establish as the incidence of ITPN is too low for proper evidence-based evaluation.

When ITPN is associated with an invasive component, it is difficult to differentiate it from adenocarcinoma. As of 2018, six cases of the local recurrence of p-ITPN were reported [9]. There is a significant difference in pathophysiology and prognosis, which makes differentiation at an earlier stage even more imperative. Pancreatic-ITPN is defined as an intraductal, grossly visible, tubule-forming epithelial neoplasm, usually with a minor papillary component, demonstrating high-grade dysplasia and ductal differentiation without mucin production [10]. Grossly, these lesions appear solid, occupying a dilated duct and without associated mucin [1]. On imaging, p-ITPN typically resembles IPMN or pancreatic adenocarcinoma with low attenuation and a dilated pancreatic duct or double duct sign. An intraductal lesion with the so-called cork in bottle sign may sometimes be detected as well.

The tumor sites for b-ITPN are intrahepatic (70\%), perihilar (20\%), and extrahepatic (10\%), and appear similar to cystic lesions or cholangiocarcinoma on imaging [5]. In the largest analysis to date of 20 patients with b-ITPN, invasive carcinoma was identified in $80 \%$ of the cases. Despite this, the survival rate was $90 \%$ for b-ITPN versus $54 \%$ for cholangiocarcinoma [5]. Similarly, an analysis of 33 patients with p-ITPN showed a 5 -year survival rate of $71 \%$ for patients without an invasive component; however, 2 of the 33 patients had an invasive cancer [11]. The pathologic diagnostic criteria for b-ITPN include growth within the intra- or extrahepatic bile duct, preinvasive histology, exophytic growth, and predominantly non-mucinous tubular units with minimal to no papillary growth [5]. In one review, the 5 -year survival rate was $80.7 \%$, compared to $37 \%$ in patients with early-stage adenocarcinoma after resection [12]. Therefore, it is important to consider this when counseling patients before surgery on an ill-defined pancreaticobiliary lesion.

Thus, how can the diagnostic accuracy of preoperative studies be improved? Perhaps a more constructive approach would be to evaluate the radiologic differences between ITPN and IPMN since there is such radiologic overlap between these two entities. Pancreatic ITPN presents a low-density lesion on pancreas-dedicated CT with a dilated pancreatic duct and potentially a "cork in bottle" sign, indicating the presence of a lesion inside the duct. MRCP is the most effective imaging modality to demonstrate this finding. One case series of p-ITPN suggested that EUS was more accurate than cross-sectional imaging [13]. Two out of the seven patients in this series had intraductal tumors identified on EUS that were not seen on CT or MRI. Despite these identifiers, atypical imaging findings such as diffuse ductal dilation can mimic chronic autoimmune pancreatitis or ductal adenocarcinoma, which may be due to long-standing disease and/or the presence of pancreatic duct stones.

Biliary-ITPN shows ductal dilation and visible intraductal soft tissue with peribiliary liver parenchyma enhancement. In b-ITPN, there is less often a dilated duct downstream given its lack of mucin production, which differentiates it from IPMN, wherein the upstream and downstream ducts are dilated, as was illustrated in our patient [14]. In a comparison of biliary IPMN and ITPN, the radiologic characteristics of ITPN were an intraductal soft tissue proportion of greater than $60 \%$, peribiliary liver parenchyma enhancement, and mild dilation of the upstream biliary system with no downstream dilation. Typically, there is less liver capsule retraction or parenchymal atrophy compared to IPMN, although in the analysis in this study these differences were not statistically significant [14]. In addition, the EUS appearance may not be characteristic and the cytologic features of the lesion without gross pathology and histologic examination may be difficult and lead to misdiagnosis.

Furthermore, as illustrated in our b-ITPN case, the diagnostic accuracy of preoperative fine-needle aspirate biopsies could be improved. In ITPN, the histologic evaluation reveals a tubulopapillary growth pattern, high-grade nuclear atypia, and the absence of mucin production [15]. Focal areas of necrosis are frequently identified, and the lesion is often attached to the duct lining by a fibrous stalk. Immunohistochemically, these neoplasms are characterized by the expression of MUC1 and MUC6 versus MUC2 and MUC5AC, which are more often found in mucinous neoplasms, consistent with our findings. While ITPNs of the pancreas and bile ducts represent premalignant lesions, they do not share molecular features with either pancreatobiliary adenocarcinomas or other premalignant lesions, such as IPMN. The most commonly reported mutations of ITPN are PIK3CA, CDKN2A/p16, and TP53, while other commonly reported mutations identified in IPMN and pancreatobiliary adenocarcinomas (KRAS, GNAS, RNF43, BRAF, EGFR, HER2, and beta-catenin) are not typically identified [1]. In this setting, the cytogenetic analysis of fine-needle aspirates may be of value in ascertaining a diagnosis preoperatively.

Finally, it is important to point out that hepatic resection for hilar cholangiocarcinoma should be limited as much as possible to achieve a curative resection. It has been suggested to perform limited hepatic resection according to the individual tumor extent of each patient [16]. Because S1 resection is known to be a prerequisite for the curative resection of hilar cholangiocarcinoma [17-19], resection of the entire S1 can be the most limited parenchymal-preserving hepatectomy in resection for hilar cholangiocarcinoma.

ITPN of the pancreas and biliary tree are rarely described entities with an elusive diagnostic workup. Resection remains 
the standard of care, with survival rates that are far superior to other potential hepatobiliary diagnoses. Herein, we discussed two illustrative cases and radiologic identifiers of these pre-malignant tumors to improve future preoperative diagnostic accuracy, which can help better prepare surgeons and patients for the eventual diagnosis.

\section{CONFLICT OF INTEREST}

No potential conflict of interest relevant to this article was reported.

\section{ORCID}

Oscar Kenneth Serrano, https://orcid.org/0000-0001-5076-0938

\section{AUTHOR CONTRIBUTIONS}

Conceptualization: AC, OKS. Methodology: All authors. Writing - original draft: JD, AC, OKS. Writing - review \& editing: JD, MB, SL, OKS.

\section{REFERENCES}

1. Kuan LL, Dennison AR, Garcea G. Intraductal tubulopapillary neoplasm of the pancreas and bile duct: a review. Pancreas 2020;49:498502.

2. Shahinian HK, Sciadini MF, Springer DJ, Reynolds VH, Lennington WJ. Tubular adenoma of the main pancreatic duct. Arch Surg 1992;127:1254-1255.

3. Park HJ, Jang KT, Heo JS, Choi YL, Han J, Kim SH. A potential case of intraductal tubulopapillary neoplasms of the bile duct. Pathol Int 2010;60:630-635.

4. Yamaguchi H, Kuboki Y, Hatori T, Yamamoto M, Shiratori K, Kawamura S, et al. Somatic mutations in PIK3CA and activation of AKT in intraductal tubulopapillary neoplasms of the pancreas. Am J Surg Pathol 2011;35:1812-1817.

5. Schlitter AM, Jang KT, Klöppel G, Saka B, Hong SM, Choi H, et al. Intraductal tubulopapillary neoplasms of the bile ducts: clinicopathologic, immunohistochemical, and molecular analysis of 20 cases. Mod Pathol 2015;28:1249-1264.

6. Visser BC, Yeh BM, Qayyum A, Way LW, McCulloch CE, Coakley FV. Characterization of cystic pancreatic masses: relative accuracy of CT and MRI. AJR Am J Roentgenol 2007;189:648-656.
7. Sainani NI, Saokar A, Deshpande V, Fernández-del Castillo C, Hahn P, Sahani DV. Comparative performance of MDCT and MRI with MR cholangiopancreatography in characterizing small pancreatic cysts. AJR Am J Roentgenol 2009;193:722-731.

8. Waters JA, Schmidt CM, Pinchot JW, White PB, Cummings OW, Pitt HA, et al. CT vs MRCP: optimal classification of IPMN type and extent. J Gastrointest Surg 2008;12:101-109.

9. Saeki K, Miyasaka Y, Ohishi Y, Yamamoto T, Matsuda R, Mochidome $\mathrm{N}$, et al. Intrapancreatic recurrence of intraductal tubulopapillary neoplasm (ITPN) 16 years after the initial surgery for noninvasive ITPN: a case report. Surg Case Rep 2018;4:96.

10. Zhang J, Ren S, Wang J, Ye D, Zhang H, Qiu W, et al. Imaging findings of intraductal tubulopapillary neoplasm (ITPN) of the pancreas: two case reports and literature review. Medicine (Baltimore) 2019;98:e14426.

11. Basturk O, Adsay V, Askan G, Dhall D, Zamboni G, Shimizu M, et al. Intraductal tubulopapillary neoplasm of the pancreas: a clinicopathologic and immunohistochemical analysis of 33 cases. Am J Surg Pathol 2017;41:313-325.

12. Date K, Okabayashi T, Shima Y, Iwata J, Sumiyoshi T, Kozuki A, et al. Clinicopathological features and surgical outcomes of intraductal tubulopapillary neoplasm of the pancreas: a systematic review. Langenbecks Arch Surg 2016;401:439-447.

13. Kim HJ, Park MS, Chung T, Kim B, Lee JH, Kim JK. Multimodality imaging studies of intraductal tubulopapillary neoplasms of the pancreas. Diagn Interv Radiol 2019;25:251-256.

14. Wu CH, Yeh YC, Tsuei YC, Huang LK, Lin CI, Liu CA, et al. Comparative radiological pathological study of biliary intraductal tubulopapillary neoplasm and biliary intraductal papillary mucinous neoplasm. Abdom Radiol (NY) 2017;42:2460-2469.

15. Kim H, Ro JY. Intraductal tubulopapillary neoplasm of the pancreas: an overview. Arch Pathol Lab Med 2018;142:420-423.

16. Nimura Y, Hayakawa N, Kamiya J, Kondo S, Shionoya S. Hepatic segmentectomy with caudate lobe resection for bile duct carcinoma of the hepatic hilus. World J Surg 1990;14:535-543; discussion 544.

17. Mizumoto R, Kawarada Y, Suzuki H. Surgical treatment of hilar carcinoma of the bile duct. Surg Gynecol Obstet 1986;162:153-158.

18. Hwang S. Standard and modified techniques for parenchyma-preserving hepatectomy focused on segments I+IV resection in patients with perihilar cholangiocarcinoma. Ann Hepatobiliary Pancreat Surg 2021;25:112-121.

19. Yang M, Li WW, Chen JH, Cui MH, Liu JL. The value of caudate lobectomy in hilar cholangiocarcinoma treatment: a meta-analysis. Medicine (Baltimore) 2021;100:e24727. 